\title{
Electromagnetics
}

\section{On the Helmholtz Theorem and Its Generalization for Multi-Layers}

\section{Alp Kustepeli}

To cite this article: Alp Kustepeli (2016) On the Helmholtz Theorem and Its Generalization for Multi-Layers, Electromagnetics, 36:3, 135-148, DOI: 10.1080/02726343.2016.1149755

To link to this article: http://dx.doi.org/10.1080/02726343.2016.1149755

曲 Published online: 13 Apr 2016.

Submit your article to this journal $₫$

III Article views: 79

Q View related articles $\sqsubset$

View Crossmark data 


\title{
On the Helmholtz Theorem and Its Generalization for Multi-Layers
}

\author{
Alp Kustepeli \\ Department of Electrical and Electronics Engineering, Izmir Institute of Technology, Izmir, Turkey
}

\begin{abstract}
The decomposition of a vector field to its curl-free and divergencefree components in terms of a scalar and a vector potential function, which is also considered as the fundamental theorem of vector analysis, is known as the Helmholtz theorem or decomposition. In the literature, it is mentioned that the theorem was previously presented by Stokes, but it is also mentioned that Stokes did not introduce any scalar and vector potentials in his expressions, which causes a contradiction. Therefore, in this article, Stokes's and Helmholtz's representations are examined in detail to reveal and emphasize their differences, similarities and important points. The Helmholtz theorem is obtained for all kinds of spaces by using the theory of distributions in a comprehensive and rigorous manner with detailed explanations, which also leads to a new surface version of the Helmholtz theorem or a new surface decomposition, resulting in the canonical form; hence, it is different than the one suggested previously in terms of two scalar functions. The generalized form of the Helmholtz theorem is also presented by employing the same approach when there is a multi-layer on the surface of discontinuity, which also corresponds to the extension of the theorem to fields with singularities of higher order.
\end{abstract}

\section{ARTICLE HISTORY}

Received 31 August 2015

Accepted 18 January 2016

\section{KEYWORDS}

Discontinuities; distribution theory; Helmholtz theorem; higher order singularities; multi-layers

\section{Introduction}

The Helmholtz theorem was introduced by Helmholtz in his 1858 paper on vortex motion. A translation of Helmholtz's paper into English was presented by Tait right after its appearance (Helmholtz, 1867). Even though it was mentioned by Tait that the translation did not pretend to be an exact one, it can be considered as a complete one since it was revised by Helmholtz. Another translation was presented by Parpart (Helmholtz, 1978). The theorem is also considered as the fundamental theorem of vector analysis of determining of a vector field of known divergence and rotation (Borisenko \& Tarapov, 1979; Sprossig, 2010). The theorem states that any vector field in space can be split into curl-free parts (irrotational or the gradient of a scalar function or potential) and divergence-free parts (solenoidal or the curl of a vector function or potential) (Morse \& Feshbach, 1953; Plonsey \& Collin, 1961; Collin, 1991; Arfken \& Weber, 1995; Sprossig, 2010; Van Bladel, 1960, 1993a, 2007, p. 1005). Vector and scalar potential representations are valid and very important in almost every field

CONTACT Alp Kustepeli alpkustepeli@iyte.edu.tr $\mathrm{B}$ Department of Electrical and Electronics Engineering, Izmir Institute of Technology, Gulbahce Koyu, 35430 Urla-Izmir, Turkey

Color versions of one or more of the figures in the article can be found online at www.tandfonline.com/uemg 
theory, and Helmholtz theorem can be used to obtain the vector and scalar potentials (Morse \& Feshbach, 1953; Kobe, 1984; Amrouche et al., 1998; Lindell \& Dassios, 2001; Kurokawa, 2001, 2008; Chubykalo et al., 2006, 2011; Chew, 2014). In the literature (Papas, 1988; Dassios \& Lindell, 2002; Zhou, 2007), it is mentioned that the theorem was previously presented by Stokes (1849), and it is also said in Zhou (2007) that Stokes did not introduce any scalar and vector potentials in his representations, resulting in an inconsistency. Therefore, in this article, Stokes's and Helmholtz's approaches and expressions are examined first to determine and present their differences.

The Helmholtz theorem is indispensable and very important in all of mathematical physics (Dassios \& Lindell, 2002), and there are some generalizations of the theorem suggested for various applications previously and very recently (Kobe, 1984; Lindell \& Dassios, 2000, 2001, 2003; Sprossig, 2010; Ortigueira et al., 2015). Physical distributions can be correctly described in terms of mathematical distributions (Schwartz, 2008, p. 77; Van Bladel, 1995, p.18), and hence, the distribution theory is very convenient for the examination of physical problems. In addition to that, the principal advantages of using a distributional approach are that distributional expressions include all space and generate and include all discontinuities in the same equation (Gagnon, 1970; İdemen, 1973; Van Bladel, 1999; Lindell, 1995). Therefore, in this study, it is also intended to employ that approach to obtain the theorem for all kinds of spaces, which may lead to a new surface version of the Helmholtz theorem or a new surface decomposition in the canonical form. The main information about the theory of distributions and its application in the electromagnetic field theory can be found in Schwartz (1950, 2008), Friedman (2005), Kanwal (2004), Friedlander and Joshi (1998), Vladimirov (2002), Taylor (1956, 1958), Gagnon (1970), Rădulet and Ciric (1971), İdemen (1973, 1990, 2011), Van Bladel (1995), and Polat $(2005,2006,2011)$.

Finally, the application of the distributional theory is investigated to obtain the most general form of the Helmholtz theorem when there is a multi-layer on the discontinuity surface or boundary, which also corresponds to the extension of the theorem to fields with singularities of higher order. The intent for the investigation of the application of the distribution theory is to obtain the Helmholtz representations without the need of confusing and complicated mathematical manipulations that might be necessary especially for problematic cases, such as higher order singularities or multi-layers.

\section{The differences and similarities between Stokes's and Helmholtz's representations}

The literature (Papas, 1988; Dassios \& Lindell, 2002; Zhou, 2007) addressed the theorem presented by Stokes (1849) and how Zhou (2007) exposed that Stokes did not introduce any scalar and vector potentials in his representations, resulting in an inconsistency. Therefore, Stokes's and Helmholtz's approaches and expressions must be examined to reveal their differences or similarities. Since Stokes's representations had been presented earlier, their examination is taken into consideration first. Stokes (1849) first examined the problems in Section I (Preliminary Analysis) of Part I 
(Theoretical Investigation) and presented the results and expressions in Subsections 5 thru 8. In Subsection 5 , the vector field $(\xi, \eta, \zeta)$, herein denoted by $\vec{F}$, which is not supposed to become infinite and to vanish at an infinite distance, was determined in infinite space if $\nabla \times \vec{F}=0$ and $\nabla \cdot \vec{F}=\delta$, where $\delta$ has finite values within a finite portion of space and vanishes elsewhere. Stokes obtained the result very elegantly by proposing that by the condition $\nabla \times \vec{F}=0, \xi d x+\eta d y+\zeta d z$ is an exact differential $d \psi$, which leads to

$$
\vec{F}=\nabla \psi
$$

where $\psi$ is a scalar potential function. By taking the divergence of $\vec{F}$, the Poisson equation is obtained as $\Delta \psi=\nabla \cdot \vec{F}$ and its solution is

$$
\psi=-\frac{1}{4 \pi} \int \frac{\nabla^{\prime} \cdot \vec{F}}{R} d \vartheta^{\prime},
$$

where $R=|\vec{R}|=\left|\vec{r}-\vec{r}^{\prime}\right|$ is the distance from observation point $\vec{r}$ to source point $\vec{r}^{\prime}$. Stokes used $r=\left|\vec{r}_{\text {Stokes }}\right|$ for that distance, but $R$ is preferred here for the sake of simplicity and $\vec{R}=-\vec{r}_{\text {Stokes. }}$. By extraordinary mathematical manipulations, Stokes also obtained two other forms for vector $\vec{F}$ as

$$
\vec{F}=-\frac{1}{4 \pi} \int \frac{\nabla^{\prime}\left(\nabla^{\prime} \cdot \vec{F}\right)}{R} d \vartheta^{\prime}
$$

and

$$
\vec{F}=\frac{1}{4 \pi} \int \nabla^{\prime} \cdot \vec{F} \frac{\hat{R}}{R^{2}} d \vartheta^{\prime},
$$

where $\hat{R}=\vec{R} / R$ is the unit vector. As a result, for vector $\vec{F}$ with the conditions $\nabla \times \vec{F}=0$ and $\nabla \cdot \vec{F} \neq 0$, Stokes presented three representations given in Eq. (1) with Eq. (2), which is the scalar potential representation, and Eq. (3) and Eq. (4). In his Subsection 6, vector field $\vec{F}$ is determined in infinite space if $\nabla \times \vec{F}=2\left(\omega^{\prime}, \omega^{\prime \prime}, \omega^{\prime \prime \prime}\right)=2 \vec{\omega}$ and $\nabla \cdot \vec{F}=0$, where $\vec{\omega}$ has finite values throughout a finite space and vanishes elsewhere. Since $\nabla \times \vec{F}=2 \vec{\omega}$, Stokes observed that $\nabla \cdot \vec{\omega}=0$, and therefore, the components of $\vec{\omega}$ are not independent. By considering $\nabla \cdot \vec{F}=0$, Stokes again obtained Poisson equation $\nabla \times \nabla \times \vec{F}=2 \nabla \times$ $\vec{\omega}=-\Delta \vec{F}$ and its solution as

$$
\vec{F}=\frac{1}{4 \pi} \int \frac{\nabla^{\prime} \times \nabla^{\prime} \times \vec{F}}{R} d \vartheta^{\prime},
$$

and in a similar manner to Eqs. (3) and (4), he wrote Eq. (5) as

$$
\vec{F}=\frac{1}{4 \pi} \int\left(\nabla^{\prime} \times \vec{F}\right) \times \frac{\vec{R}}{R^{3}} d \vartheta^{\prime} .
$$

As a result, for vector $\vec{F}$ with conditions $\nabla \times \vec{F} \neq 0$ and $\nabla \cdot \vec{F}=0$, Stokes presented two representations, given in Eqs. (5) and (6), which do not include a vector potential representation as in the Helmholtz theorem. One can certainly obtain the form 


$$
\vec{F}=\nabla \times\left(\frac{1}{4 \pi} \int \frac{\nabla^{\prime} \times \vec{F}}{R} d \vartheta^{\prime}\right)
$$

leading to the vector potential representation $\vec{F}=\nabla \times \vec{A}$ from Stokes's representations by mathematical manipulations, especially if those in his article, presented here from Eqs. (1) to (3), are taken into consideration; it can hence be considered that the vector potential representation is implicit in the equations, but it was not given explicitly by Stokes. In his Subsection 7, Stokes combined the above results and added the expressions to obtain a vector field $\vec{F}$ satisfying conditions $\nabla \cdot \vec{F} \neq 0$ and $\nabla \times \vec{F} \neq 0$. Therefore, vector field $\vec{F}$ is obtained by any combination of the three expressions given in Eqs. (1), (3) and (4) with the two given in Eqs. (5) and (6). One can also conclude from the above results that Stokes used a scalar potential representation for the curl-free (irrotational) part, but he did not use a vector potential representation for the divergence-free (solenoidal) part. Even though Stokes used a scalar potential function for the curl-free part, he did not propose the usage of that function at the beginning and obtained it as a result. In his Subsection 8 , Stokes decomposed a vector field $\vec{F}$ into its curl-free and divergence-free parts by first obtaining the curl-free part and subtracting it from the vector $\vec{F}$ itself, which certainly gives the decomposition and the divergencefree part, but again not in a vector potential form.

When the famous article of Helmholtz (1858) is examined, it is seen that Helmholtz's motivation and handling is actually very different. Helmholtz examined the problem of finding the vector $(u, v, w)$, which is again denoted by $\vec{F}$ here, if $\nabla \times \vec{F}=-2(\xi, \eta, \zeta)$ is known and $\nabla \cdot \vec{F}=0$ in a bounded region $S$, not in an infinite space, in other words, obtaining vector $\vec{F}$ with the conditions $\nabla \times \vec{F} \neq 0$ and $\nabla \cdot \vec{F}=0$ in a finite region. On p. 38 of his article, Helmholtz claimed that the solution can be obtained in terms of a vector function $\vec{A}$ and up to an additive gradient of a scalar function $P$ in a general form of

$$
\vec{F}=\nabla P+\nabla \times \vec{A}
$$

The gradient of an arbitrary scalar function $P$ can be added since $\nabla \times \nabla P=0$, and if $\nabla \cdot \vec{A}=0$, which he proved later by taking into consideration $\nabla \cdot(\nabla \times \vec{F})=0$ in the region and $\hat{n} \cdot(\nabla \times \vec{F})=0$ over the whole boundary where $\hat{n}$ is the unit normal vector, Eq. (8) leads to the Poisson equation $\Delta \vec{A}=-\nabla \times \vec{F}$ for the vector function $\vec{A}$, and its solution is obvious. Since $\nabla \cdot \vec{F}=0$ in the finite region, Helmholtz obtained the Laplace equation $\Delta P=0$ for scalar function $P$. As a conclusion, Helmholtz employed scalar funtion $P$ to satisfy the boundary conditions with its gradient. Function $P$ can be determined by using and integrating another arbitrary function $k$ due to an external mass $X$. In his article, Helmholtz (1858) availed himself of the analogies of fluids and electromagnetism, and therefore, it can be concluded that $k$ can be thought of as $-\nabla \cdot \vec{F} /(4 \pi)$ and the Poisson equation for $P$ is $\Delta P=\nabla \cdot \vec{F}$ in the whole space, even if it is not written explicitly, and since all the integrals are extended to the exterior of the finite region to cover the boundary conditions, final formulation is also considered to be valid for all space as 


$$
\vec{F}=-\nabla\left(\frac{1}{4 \pi} \int \frac{\nabla^{\prime} \cdot \vec{F}}{R} d \vartheta^{\prime}\right)+\nabla \times\left(\frac{1}{4 \pi} \int \frac{\nabla^{\prime} \times \vec{F}}{R} d \vartheta^{\prime}\right)
$$

which is the very well-known expression of the Helmholtz theorem.

As can be seen from the above discussions, there are actually different aspects of the two representations, and moreover, the expressions for the divergence-free (solenoidal) part given by Stokes and Helmholtz have different representations, but one can certainly obtain one from the other by mathematical manipulations. At that point, it is beneficial to repeat the general comment made by Tait about the works of Stokes and Helmholtz at the end of his translation (Helmholtz, 1867, p. 511):

A portion of the contents of the paper had been anticipated by Professor Stokes in various excellent papers in the Cambridge Philosophical Transactions; but the discovery of the nature and motions of vortex-filaments is entirely novel, and of great consequence.

The representations presented by Stokes and Helmholtz might be considered to be in a less complete form, especially in terms of uniqueness (Papas, 1988; Lindell \& Dassios, 2000, 2001). A comprehensive and rigorous examination of the representations was presented by Blumenthal (1905), including the uniqueness. Blumenthal's expressions (Blumenthal, 1905; Van Bladel, 1993a) are very similar, in a sense, to both of those presented by Stokes and Helmholtz. Moreover, Blumenthal mentioned that the expressions are also valid in the plane if $1 /\left|\vec{r}-\vec{r}^{\prime}\right|$ in the equations is replaced by $\log \left|\vec{r}-\vec{r}^{\prime}\right|$. Additional discussions about the uniqueness and existence as well can be seen in Morse and Feshbach (1953), Van Bladel (1960), Borisenko and Tarapov (1979), Arfken and Weber (1995), and Dassios and Lindell (2002).

\section{The Helmholtz theorem for all kinds of spaces by distributional approach}

As seen from the previous section, the Helmholtz theorem is obtained by using the potential theory via the scalar and vector Poisson equations, which is always the main approach in various methods used to determine the representations for the theorem in infinite and finite spaces as well. Morse and Feshbach (1953) used the vector identity and found the solution of the vector Poisson equation in $\vec{F}=$ $-\Delta \vec{W}=-\nabla(\nabla \cdot \vec{W})+\nabla \times(\nabla \times \vec{W})=\nabla \varphi+\nabla \times \vec{A}$ to obtain the theorem in finite and infinite spaces. Plonsey and Collin (1961) used the integration of the Poisson equation, with the properties of $\nabla(1 / R)$ and $\Delta(1 / R)$, except the singular point $R=0$, and mathematical manipulations similar to those in Morse and Feshbach (1953) for the representation of the theorem in finite space. Collin (1991) used a similar method to Plonsey and Collin (1961) with the only difference of using the delta function property of $\Delta(1 / R)$. Van Bladel (1993a, 2007, p. 1005) used the derivative operators in the sense of distributions in the representation of the theorem in infinite space to obtain the representations in finite space or for piecewise continuous vectors. The last two can also be considered as the special applications of the theory of distributions. There were also some other attempts (Zhou, 2007; Gui \& Dou, 2007) to obtain the theorem for discontinuous vector fields, such as the one presented by Kurokawa (2001), but if the field components are discontinuous, their derivatives become infinite and the differential forms are not valid everywhere in the classical 
sense. Therefore, mathematical details are very important and the representations must be given accordingly and rigorously (İdemen, 1973; Lindell, 1995; Kurokawa, 2001).

The Helmholtz theorem can be associated with fields and sources resulting in discontinuities as well (Plonsey \& Collin, 1961; Lindell \& Dassios, 2001), and since physical distributions can be correctly described in terms of mathematical distributions (Schwartz, 2008, p. 77; Van Bladel, 1995, p. 18), the distribution theory is very convenient for the examination of physical problems. In addition, the principal advantages of using a distributional approach are that distributional expressions include all space and generate and include all discontinuities in the same equation (Gagnon, 1970; İdemen, 1973; Van Bladel, 1999; Lindell, 1995). Moreover, the Poisson equation used to determine the Helmholtz representations is valid in the sense of distributions throughout the whole space (Schwartz, 2008, p. 122; Van Bladel, 1995, p. 22). Therefore, it might be beneficial to consider the comprehensive and rigorous investigation of the application of the distribution theory to obtain the Helmholtz theorem, especially for the usage or extension of the theorem in complicated cases, such as multi-layers or fields with singularities of higher order.

If the distributional form of the Poisson equation

$$
\Delta V=-\rho / \varepsilon_{0}
$$

is considered, where $V$ is the potential distribution, $\rho$ is the volume charge density, and $\varepsilon_{0}$ is the permittivity of free space, the solution is obtained as

$$
V(\vec{r})=\rho * \frac{1}{4 \pi \varepsilon_{0} r}=\frac{1}{4 \pi \varepsilon_{0}} \int \frac{\rho\left(\vec{r}^{\prime}\right)}{\left|\vec{r}-\vec{r}^{\prime}\right|} d \vartheta^{\prime},
$$

the convolution of the two distributions, the charge distribution $\rho$, and the distribution $1 /\left(4 \pi \varepsilon_{0} r\right)$, where $r=|\vec{r}|$ is the distance from the origin to the point $\vec{r}$ (Schwartz, 2008, p. 122; Gagnon, 1970; Kanwal, 2004, p. 197; Vladimirov, 2002, p. 68). As can be seen in Schwartz (2008, p. 117), the convolution in Eq. (11) actually has the meaning

$$
\left\langle\rho\left(\vec{r}^{\prime}\right), \frac{1}{4 \pi \varepsilon_{0}\left|\vec{r}-\vec{r}^{\prime}\right|}\right\rangle
$$

by considering $1 /\left(4 \pi \varepsilon_{0}\left|\vec{r}-\vec{r}^{\prime}\right|\right)$ as a function of only $\vec{r}^{\prime}$, which is in $D$ representing the space of test functions with compact support, where $\vec{r}$ is fixed. \langle\rangle is defined by

$$
\langle f, t\rangle=\int f t d \vartheta
$$

where $f$ is a distribution, $t$ is a test function, and the integral sign without the limits indicates that the integration is extended over all space (Schwartz, 2008, p. 77; Gagnon, 1970; Kanwal, 2004, p. 22; Friedman, 2005, chap. 2; Van Bladel, 1995, p. 6, 2007, p. 1090). Even though $t$ is considered in $D$, as mentioned by Schwartz (2008, p. 77), many expressions as in Eqs. (12) and (13) and must be evaluated in physical problems, although $t$ does not in general belong to $D$, and each distribution $f$ can be extended as a functional over a set of functions larger than $D$, and the test functions are not required to have compact support, e.g., the space $E$ (Kanwal, 2004, sec. 7.6). In addition to that, from the discussion presented in Kanwal (2004, Sec. 7.6), it is seen that the test functions of compact support lead to the distributions of arbitrary 
support, whereas the test functions of arbitrary support yield the distributions of compact support and Cauchy representation of a distribution, which is very similar to Eq. (12), is presented as an example for that case. One can appreciate these remarks if the differences between the generalized functions and distributions are examined (Friedman, 2005, chaps. 2 and 3). Even though they are often used interchangeably in the literature, many theorems that are valid for distributions do not have analogies for arbitrary generalized functions, but they can still be extended to some classes of generalized functions (Friedman, 2005, chap. 2). As can also be seen in Friedman (2005, chap. 3) that for generalized functions, test functions do not need to have a compact support, and a test function that has a compact support is called a finite test function. In addition to that, it is seen that the definition of a derivative for a distribution fits with that of a generalized function (Friedman, 2005, pp. 36 and 52), leading to the well-known formulation

$$
\langle\Delta f, t\rangle=\langle f, \Delta t\rangle,
$$

which can be obtained for both cases. The solution of the Poisson equation given in Eq. (11) can also be obtained by considering Eq. (14) in primed coordinates with Eq. (10) in a similar manner to Eq. (12), $t=1 / R$ and the Laplacian of $t$ with respect to the primed coordinates, which is $\Delta^{\prime} t=\Delta^{\prime}(1 / R)=\Delta(1 / R)=-4 \pi \delta\left(\vec{r}-\vec{r}^{\prime}\right)$.

By employing the distribution theory and the above information, one can easily obtain the Helmholtz theorem in infinite space and in finite space or for piecewise continuous vector fields in the canonical form

$$
\vec{F}=-\nabla V+\nabla \times \vec{A}
$$

by considering the scalar and vector Poisson equations in the sense of distributions, obtained in terms of $V$ and $\vec{A}$ as

$$
\Delta V=-\nabla \cdot \vec{F}
$$

and

$$
\Delta \vec{A}=-\nabla \times \vec{F}
$$

respectively, where $\nabla \cdot \vec{A}=0$. By obtaining the solutions of Eqs. (16) and (17) and using the distributional approach presented above and Eqs. (10) thru (15), the vector $\vec{F}$ can also be expressed as Eq. (9).

The benefit of employing the theory of distributions is more appreciable if one considers the discontinuous field components and their derivatives that become infinite. For a piecewise continuous vector field in the whole space, the Helmholtz theorem or decomposition can be obtained by taking into consideration the distributional forms of divergence and curl (Gagnon, 1970; Rădulet \& Ciric, 1971; İdemen, 1973; Van Bladel, 1999),

$$
\begin{aligned}
\nabla \cdot \vec{F} & =\{\nabla \cdot \vec{F}\}+\hat{n} \cdot[[\vec{F}]] \delta(S), \\
\nabla \times \vec{F} & =\{\nabla \times \vec{F}\}+\hat{n} \times[[\vec{F}]] \delta(S),
\end{aligned}
$$

in Eqs. (16) and (17), respectively, where $S$ is the surface of discontinuity, which separates the two contiguous regions $\vartheta_{1}$ and $\vartheta_{2}$, where $\vec{r} \notin S$. If a vector field considered 
in those regions is represented as $\vec{F}=\{\vec{F}\},\{\}$ will later be called the regular part of the quantity considered, its jump across $S$ is denoted by $[[\vec{F}]]=\vec{F}^{2}-\vec{F}^{1}$ in Eqs. (18) and (19), where $\vec{F}^{2}$ and $\vec{F}^{1}$ are the boundary values of $\vec{F}$ on $S$ from $\vartheta_{2}$ and $\vartheta_{1}$, respectively. The unit normal vector of the surface is again denoted by $\hat{n}$, and it is directed into $\vartheta_{2} . \delta(S)$ denotes the Dirac delta distribution concentrated on $S$. In addition to Eqs. (18) and (19), many other useful distributional relations can be found in Gagnon (1970). Finally one obtains

$$
\{\vec{F}\}=-\nabla\left(\int \frac{\left\{\nabla^{\prime} \cdot \vec{F}\right\}+\hat{n}^{\prime} \cdot[[\vec{F}]] \delta(S)}{4 \pi R} d \vartheta^{\prime}\right)+\nabla \times\left(\int \frac{\left\{\nabla^{\prime} \times \vec{F}\right\}+\hat{n}^{\prime} \times[[\vec{F}]] \delta(S)}{4 \pi R} d \vartheta^{\prime}\right),
$$

and the volume integrals can be replaced by the surface integrals due to $\delta(S)$, resulting in

$$
\{\vec{F}\}=-\nabla\left(\int \frac{\left\{\nabla^{\prime} \cdot \vec{F}\right\}}{4 \pi R} d \vartheta^{\prime}+\int_{S} \frac{\hat{n}^{\prime} \cdot[[\vec{F}]]}{4 \pi R} d S^{\prime}\right)+\nabla \times\left(\int \frac{\left\{\nabla^{\prime} \times \vec{F}\right\}}{4 \pi R} d \vartheta^{\prime}+\int_{S} \frac{\hat{n}^{\prime} \times[[\vec{F}]]}{4 \pi R} d S^{\prime}\right),
$$

which is the Helmholtz theorem in the whole space for a piecewise continuous vector field $\vec{F}=\{\vec{F}\}$, and it can be considered as the fundamental expression to obtain the Helmholtz theorem for all kinds of spaces, such as those in Van Bladel (1960, 2007, p. 1008), Kurokawa (2001, 2008), Zhou (2007) and, Gui and Dou (2007). The Helmholtz theorem in a finite space or in a closed region $\vartheta_{1}=\vartheta$ and its boundary denoted by $\partial \vartheta=S$ can be obtained from Eq. (21). $\vec{F}$ can be chosen to be the vector field inside $\vartheta$ and be zero outside of it, then $[[\vec{F}]]=0-\vec{F}=-\vec{F}$ on $S$, which is only a function of surface coordinates, if Eq. (21) is rewritten to

$$
\vec{F}=-\nabla\left(\int_{\vartheta} \frac{\nabla^{\prime} \cdot \vec{F}}{4 \pi R} d \vartheta^{\prime}-\oint_{\partial \vartheta=S} \frac{\hat{n}^{\prime} \cdot \vec{F}}{4 \pi R} d S^{\prime}\right)+\nabla \times\left(\int_{\vartheta} \frac{\nabla^{\prime} \times \vec{F}}{4 \pi R} d \vartheta^{\prime}-\oint_{\partial \vartheta=S} \frac{\hat{n}^{\prime} \times \vec{F}}{4 \pi R} d S^{\prime}\right),
$$

which is the Helmholtz theorem in a finite region (Morse \& Feshbach, 1953; Plonsey \& Collin, 1961; Collin, 1991; Van Bladel, 1993a, 2007, p. 1007). As a result, it is seen that the Helmholtz theorem can easily be obtained by a distributional approach for all kinds of spaces and discontinuities.

\section{A new surface version of the Helmholtz theorem}

In view of the Helmholtz theorem, an attempt to obtain the decomposition for the special case of the surface electric current on a conducting body can be seen in Scharstein (1991). In that study, the representation for the decomposition was proposed in terms of two scalar functions as $\vec{J}_{S}=\nabla_{S} \Phi+\nabla_{S} \times(\hat{n} \Omega)$, which seems to be equivalent to that given by Van Bladel (1993b, 1993c, 2007, p. 1008) as $\vec{J}_{S}=\nabla_{S} \phi+\hat{n} \times \nabla_{S} \theta$ with $\Phi=\phi$ and $\Omega=-\theta$, where $\nabla_{S}$ represents the surface gradient given by Van Bladel (2007, p. 1027). Tai (1992, p. 80) used another approach and presented a different representation for the surface gradient; this study uses Van Bladel's treatment, which is the classical approach for the surface operators given by Weatherburn (1955). Even though the two representations and Poisson equations to be used for the solution 
of the two scalar functions appear to be the same, one must be careful about the interpretation, expression, and usage of the surface curl presented for a tangential surface field by Eq. (7) in Scharstein (1991). Since the vector $\hat{n} \Omega$ is not a tangential surface field and is perpendicular to the contour of the integral on the surface, the term $\nabla_{S} \times(\hat{n} \Omega)$ in the decomposition can be thought to be zero with that definition, which may result in an inconsistency.

After using the theory of distributions in the previous section to obtain the Helmholtz theorem in infinite or finite spaces, and since distributional expressions include all space, it is now intended to employ the same approach to obtain the surface version of the Helmholtz theorem or surface decomposition in the canonical form as well, using a scalar and a vector potential function, which is therefore different than those with the above-mentioned two scalar functions. A general surface vector field $\vec{F}$ to be considered on a surface $S$ can be expressed in the sense of distributions as $\vec{F}=\vec{F}_{0} \delta(S)$, and it is obvious that $\{\vec{F}\}=0$. To obtain the representation, one must first obtain the divergence and curl of that vector. Considering $\nabla \delta(S) \cdot \hat{n}=\partial \delta(S) / \partial n$, or in general $\nabla \delta^{(k)}(S)=\hat{n} \delta^{(k+1)}(S)$, which can be obtained by combining the Cartesian derivatives presented in Eq. (5.4.8) of Kanwal (2004, p. 112), where $\delta^{(k)}(S)$ denotes the normal derivative of the Dirac delta distribution of arbitrary order $k \geq 0$, the identities for scalar and vector distributions given in Gagnon (1970), and taking into account that $\vec{F}_{0}$ may only be a function of surface coordinates in the Dupin coordinate system (Van Bladel, 2007, p. 1027; Tai, 1992, p. 15), give

$$
\begin{aligned}
\nabla \cdot \vec{F} & =\nabla \cdot\left(\vec{F}_{0} \delta(S)\right) \\
& =\left(\nabla \cdot \vec{F}_{0}\right) \delta(S)+\nabla \delta(S) \cdot \vec{F}_{0} \\
& =\left(\nabla_{S} \cdot \vec{F}_{0}\right) \delta(S)+\hat{n} \cdot \vec{F}_{0} \delta^{(1)}(S),
\end{aligned}
$$

and similarly,

$$
\begin{aligned}
\nabla \times \vec{F} & =\nabla \times\left(\vec{F}_{0} \delta(S)\right) \\
& =\left(\nabla_{S} \times \vec{F}_{0}\right) \delta(S)+\hat{n} \times \vec{F}_{0} \delta^{(1)}(S) .
\end{aligned}
$$

Considering Eqs. (23) and (24) in Eqs. (16) and (17), respectively, gives

$$
\begin{aligned}
\vec{F}_{0}= & -\nabla\left(\frac{1}{4 \pi} \int \frac{\left(\nabla^{\prime}{ }_{S} \cdot \vec{F}_{0}\right) \delta(S)+\hat{n}^{\prime} \cdot \vec{F}_{0} \delta^{(1)}(S)}{R} d \vartheta^{\prime}\right) \\
& +\nabla \times\left(\frac{1}{4 \pi} \int \frac{\left(\nabla^{\prime}{ }_{S} \times \vec{F}_{0}\right) \delta(S)+\hat{n}^{\prime} \times \vec{F}_{0} \delta^{(1)}(S)}{R} d \vartheta^{\prime}\right)
\end{aligned}
$$

in which the normal derivative of the Dirac delta distribution $\delta^{(1)}(S)=\partial \delta(S) / \partial n$ is seen. By employing the distributional approach, it becomes possible to easily transfer the normal derivative to test function $t$. One might consider

$$
\left\langle\frac{\partial \delta(S)}{\partial n}, t\right\rangle=-\left\langle\delta(S), \frac{\partial t}{\partial n}\right\rangle
$$


for the transfer as given in the literature (Schwartz, 2008, p. 88; Van Bladel, 1995, p. 19, 1999, 2007, p. 1097; Vladimirov, 2002, p. 30), but Eq. (26) is not correct and

$$
\left\langle\frac{\partial \delta(S)}{\partial n}, t\right\rangle \neq-\left\langle\delta(S), \frac{\partial t}{\partial n}\right\rangle .
$$

The correct form is given by Kanwal (2004, p. 109) as

$$
\left\langle\frac{\partial \delta(S)}{\partial n}, t\right\rangle=-\left\langle\delta(S), \frac{\partial t}{\partial n}-J t\right\rangle,
$$

implying

$$
\left\langle\delta^{(k)}(S), t\right\rangle=(-1)^{k}\left\langle\delta(S),\left(\frac{\partial}{\partial n}-J\right)^{k} t\right\rangle,
$$

which will be used later for the generalization, where $J=1 / R_{1}+1 / R_{2}$ is the first curvature of the surface of discontinuity $S$ with $R_{1}$ and $R_{2}$ being the two principal radii of curvature (Van Bladel, 2007, p. 1026). Considering Eq. (28) and $t=1 / R$ according to the discussions presented in the previous section, Eq. (25) can be rewritten to

$$
\begin{aligned}
\vec{F}_{0}= & -\nabla\left(\int_{S} \frac{\nabla_{S}^{\prime} \cdot \vec{F}_{0}}{4 \pi R} d S^{\prime}-\int_{S} \frac{\hat{n}^{\prime} \cdot \vec{F}_{0}}{4 \pi}\left[\left(\frac{\partial}{\partial n^{\prime}}-J\right) \frac{1}{R}\right] d S^{\prime}\right) \\
& +\nabla \times\left(\int_{S} \frac{\nabla_{S}^{\prime} \times \vec{F}_{0}}{4 \pi R} d S^{\prime}-\int_{S} \frac{\hat{n}^{\prime} \times \vec{F}_{0}}{4 \pi}\left[\left(\frac{\partial}{\partial n^{\prime}}-J\right) \frac{1}{R}\right] d S^{\prime}\right),
\end{aligned}
$$

which is the surface version of the Helmholtz theorem or surface decomposition for a general surface vector field $\vec{F}=\vec{F}_{0} \delta(S)$; it is obtained in the canonical form as well. If $\vec{F}_{0}$ is a tangential vector function as in the case of a surface current density $\vec{J}=\vec{J}_{S} \delta(S)$, the second integral in the gradient operator in Eq. (30) vanishes since $\hat{n} \cdot \vec{J}_{S}=0$, and one can easily obtain the surface decomposition for a surface current. In that case, the part with the gradient in Eq. (30) corresponds to the longitudinal term given in Van Bladel (1993b), but the part with the curl in Eq. (30) is different than the transverse term; therefore, even if it is considered for a tangential surface vector, the representation presented in Eq. (30) can be considered as a new surface version of the Helmholtz theorem or a new surface decomposition when it is compared with the one suggested previously in terms of two scalar functions (Scharstein, 1991; Van Bladel, 1993b, 1993c, 2007, p. 1008).

\section{Generalization of the Helmholtz theorem for multi-layers}

In the previous two sections, the Helmholtz representations for the vector fields $\vec{F}=$ $\{\vec{F}\}$ in spaces and $\vec{F}=\vec{F}_{0} \delta(S)$ on surfaces, which represents a single layer on $S$, were obtained by using a distributional approach. From the linearity of the equations, it is obvious that the Helmholtz representation can easily be obtained for a vector field $\vec{F}=$ $\{\vec{F}\}+\vec{F}_{0} \delta(S)$ by adding the expressions obtained in the last two sections, namely 
those given in Eqs. (21) and (30), accordingly. Considering these results and a theorem that a distribution with compact support has a finite order (Friedman, 2005, chap. 3; Friedlander \& Joshi, 1998, chap. 3; Vladimirov, 2002, p. 36; Polat, 2011), it can be concluded that the general distributional representation including multi-layers can be given as

$$
\vec{F}=\{\vec{F}\}+\sum_{k=0}^{N} \vec{F}_{k} \delta^{(k)}(S)
$$

for a vector. In Eq. (31), $\{\vec{F}\}$ denotes the regular part as mentioned previously, and the term with the summation represents the singular part of the distribution, which consists of the linear combination of the Dirac delta distribution $\delta(S)$ and its normal derivatives $\delta^{(k)}(S)$ concentrated on the surface of discontinuity $S$. The singular part on $S$ might be denoted by $[\vec{F}]_{S} . N$ is the order of the distribution and is finite due to the theorem. The information about the generalized forms of the Poisson equation and its solution for the most general case including multi-layers can be found in Kustepeli (2015). It is now intended to obtain the most general form of the Helmholtz theorem when there is also a multi-layer on the discontinuity surface or in the presence of higher order singularities. In that case, since the Helmholtz representations were obtained for $\vec{F}=\{\vec{F}\}$ and $\vec{F}=\vec{F}_{0} \delta(S)$, to obtain the generalized Helmholtz theorem, it will be enough to find the Helmholtz representations for each $\vec{F}=\vec{F}_{k} \delta^{(k)}(S)$ on surface $S$. Taking the divergence and curl similar Eqs. (23) and (24) gives

$$
\begin{gathered}
\nabla \cdot\left(\vec{F}_{k} \delta^{(k)}(S)\right)=\left(\nabla_{S} \cdot \vec{F}_{k}\right) \delta^{(k)}(S)+\hat{n} \cdot \vec{F}_{k} \delta^{(k+1)}(S), \\
\nabla \times\left(\vec{F}_{k} \delta^{(k)}(S)\right)=\left(\nabla_{S} \times \vec{F}_{k}\right) \delta^{(k)}(S)+\hat{n} \times \vec{F}_{k} \delta^{(k+1)}(S),
\end{gathered}
$$

and the representation can be obtained similar to Eqs. (25) and (30), considering Eq. (29), as

$$
\begin{aligned}
\vec{F}_{k} \delta^{(k)}(S)= & -\nabla\left((-1)^{k} \int_{S} \frac{\nabla_{S}^{\prime} \cdot \vec{F}_{k}}{4 \pi}\left[\left(\frac{\partial}{\partial n^{\prime}}-J\right)^{k} \frac{1}{R}\right] d S^{\prime}+(-1)^{k+1} \int_{S} \frac{\hat{n}^{\prime} \cdot \vec{F}_{k}}{4 \pi}\left[\left(\frac{\partial}{\partial n^{\prime}}-J\right)^{k+1} \frac{1}{R}\right] d S^{\prime}\right) \\
& +\nabla \times\left((-1)^{k} \int_{S} \frac{\nabla_{S}^{\prime} \times \vec{F}_{k}}{4 \pi}\left[\left(\frac{\partial}{\partial n^{\prime}}-J\right)^{k} \frac{1}{R}\right] d S^{\prime}+(-1)^{k+1} \int_{S} \frac{\hat{n}^{\prime} \times \vec{F}_{k}}{4 \pi}\left[\left(\frac{\partial}{\partial n^{\prime}}-J\right)^{k+1} \frac{1}{R}\right] d S^{\prime}\right)
\end{aligned}
$$

for $k=0,1, \ldots, N$ on surface $S$. Finally, the generalized Helmholtz theorem or decomposition for $\vec{F}$ given in Eq. (31) can be obtained by using the expressions for its regular part $\{\vec{F}\}$ given by Eq. (21) and its singular part represented by Eq. (34). The Helmholtz representation for the singular part $[\vec{F}]_{S}$ on the surface of discontinuity $S$ can be obtained by adding $\vec{F}_{k} \delta^{(k)}(S)$ presented in Eq. (34) for $k=0,1, \ldots, N$ accordingly, which might be written as 


$$
\begin{aligned}
{[\vec{F}]_{S}=} & -\nabla\left(\int_{S} \frac{\nabla_{S}^{\prime} \cdot \vec{F}_{0}}{4 \pi R} d S^{\prime}\right. \\
& +\sum_{k=1}^{N}(-1)^{k} \int_{S} \frac{\nabla_{S}^{\prime} \cdot \vec{F}_{k}+\hat{n}^{\prime} \cdot \vec{F}_{k-1}}{4 \pi}\left[\left(\frac{\partial}{\partial n^{\prime}}-J\right)^{k} \frac{1}{R}\right] d S^{\prime} \\
& \left.+(-1)^{N+1} \int_{S} \frac{\hat{n}^{\prime} \cdot \vec{F}_{N}}{4 \pi}\left[\left(\frac{\partial}{\partial n^{\prime}}-J\right)^{N+1} \frac{1}{R}\right] d S^{\prime}\right) \\
& +\nabla \times\left(\int_{S} \frac{\nabla_{S}^{\prime} \times \vec{F}_{0}}{4 \pi R} d S^{\prime}\right. \\
& +\sum_{k=1}^{N}(-1)^{k} \int_{S} \frac{\nabla_{S}^{\prime} \times \vec{F}_{k}+\hat{n}^{\prime} \times \vec{F}_{k-1}}{4 \pi}\left[\left(\frac{\partial}{\partial n^{\prime}}-J\right)^{k} \frac{1}{R}\right] d S^{\prime} \\
& \left.+(-1)^{N+1} \int_{S} \frac{\hat{n}^{\prime} \times \vec{F}_{N}}{4 \pi}\left[\left(\frac{\partial}{\partial n^{\prime}}-J\right)^{N+1} \frac{1}{R}\right] d S^{\prime}\right) .
\end{aligned}
$$

\section{Conclusion}

In this article, Stokes's and Helmholtz's representations have been reviewed in detail to reveal their differences and similarities. The Helmholtz theorem has been obtained for all kinds of spaces by using the theory of distributions in a comprehensive and rigorous manner, which has also led to a new surface version of the Helmholtz theorem or surface decomposition, resulting in the canonical form, and therefore, it is different than the one suggested previously in terms of two scalar functions. By employing the same approach, the extension of the Helmholtz theorem has been presented when there is a multi-layer on the surface of discontinuity, which corresponds to fields with singularities of higher order.

\section{References}

Amrouche, C., C. Bernardi, M. Dauge, \& V. Girault. 1998. Vector potentials in three-dimensional non-smooth domains. Math. Meth. Appl. Sci. 21:823-864.

Arfken, G. B., \& H. J. Weber. 1995. Mathematical Methods for Physicists, 4th ed., Sec. 1.16. San Diego: Academic Press.

Blumenthal, O. 1905. Über die Zerlegung unendlicher Vektorfelder (On the decomposition of infinite vector fields). Math. Annalen 61:235-250.

Borisenko, A. I., \& I. E. Tarapov. 1979. Vector and tensor analysis with application, Sec. 5.7. New York: Dover.

Chew, W. C. 2014. Vector potential electromagnetics with generalized gauge for inhomogeneous media: Formulation. PIER 149:69-84.

Chubykalo, A., A. Espinoza, R. Alvarado Flores, \& A. Gutierrez Rodriguez. 2006. Helmholtz theorem and the v-gauge in the problem of superluminal and instantaneous signals in classical electrodynamics. Found. Phys. Lett. 19:37-49.

Chubykalo, A., A. Espinoza, \& R. Alvarado Flores. 2011. Electromagnetic potentials without gauge transformations. Phys. Scr. 84:1-6.

Collin, R. E. 1991. Field theory of guided waves, 2nd ed., 798-801. New York: IEEE Press. 
Dassios, G., \& I. V. Lindell. 2002. Uniqueness and reconstruction for the anisotropic Helmholtz decomposition. J. Phys. A: Math. Gen. 35:5139-5146.

Friedlander, F. G., \& M. Joshi. 1998. Introduction to the theory of distributions. Cambridge, UK: Cambridge University Press.

Friedman, A. 2005. Generalized functions and partial differential equations. New York, NY: Dover.

Gagnon, R. J. 1970. Distribution theory of vector fields. Am. J. Phys. 38:879-891.

Gui, Y. F., \& W. B. Dou. 2007. A rigorous and completed statement on Helmholtz theorem. PIER 69:287-304.

Helmholtz, H. L. F. 1858. Über Integrale der hydrodynamischen Gleichungen, welcheden Wirbelbewegungen entsprechen. J. Reine Angew. Math. 55:25-55.

Helmholtz, H. L. F. 1867. On integrals of the hydrodynamical equations, which express vortexmotion. Phil. Mag. 33: 485-512(translated by P. G. Tait).

Helmholtz, H. L. F. 1978. On integrals of the hydrodynamic equations that correspond to vortexmotion. Int. J. Fusion Energy 1:41-68 (translated by U. Parpart).

İdemen, M. 1973. The Maxwell equations in the sense of distributions. IEEE Trans. Antennas Propagat. 21:736-738.

İdemen, M. 1990. Universal boundary relations of the electromagnetic field. J. Phys. Soc. Japan 59:71-80.

İdemen, M. 2011. Discontinuities in the electromagnetic field. New York: IEEE Press.

Kanwal, R. P. 2004. Generalized functions, 3rd ed. Boston: Birkhäuser.

Kobe, D. H. 1984. Helmholtz theorem for antisymmetric second-rank tensor fields and electromagnetism with magnetic monopoles. Am. J. Phys. 52:354-358.

Kurokawa, K. 2001. General expressions for vector and scalar potentials. IEEE Trans. Antennas Propagat. 49:1315-1321.

Kurokawa, K. 2008. Vector potentials in simply structured spaces. IEEE Trans. Antennas Propagat. 56:976-980.

Kustepeli, A. 2015. Revised distributional forms of the Laplacian and Poisson's equation, their implications and all related generalizations. Electromagnetics 35:371-385.

Lindell, I. V. 1995. Methods for electromagnetic field analysis, 69-70. New York: IEEE Press.

Lindell, I. V., \& G. Dassios. 2000. Generalized Helmholtz decomposition and static electromagnetics. JEMWA 14:1415-1428.

Lindell, I. V., \& G. Dassios. 2001. The Helmholtz theorem and scalar potential expansion. JEMWA 15:1281-1295.

Lindell, I. V., \& G. Dassios. 2003. Helmholtz theorem for multiform fields. JEMWA 17:3-14.

Morse, P. M., \& H. Feshbach. 1953. Methods of theoretical physics, 52-54. New York: McGraw-Hill.

Ortigueira, M. D., M. Rivero, \& J. J. Trujillo. 2015. From a generalized Helmholtz decomposition theorem to fractional Maxwell equations. Commun. Nonlinear Sci. Numer. Simulat. 22:1036-1049.

Papas, C. H. 1988. Theory of electromagnetic wave propagation, 9. New York: Dover.

Plonsey, R., \& R. E. Collin. 1961. Principles and applications of electromagnetic fields, Sec. 1.18. New York: McGraw-Hill.

Polat, B. 2005. Remarks on the fundamental postulates on field singularities in electromagnetic theory. IEEE Antennas Propagat. Mag. 47:47-54.

Polat, B. 2006. A note regarding "Remarks on the fundamental postulates on field singularities in electromagnetic theory". IEEE Antennas Propagat. Mag. 48:105.

Polat, B. 2011. Approximate boundary relations on anisotropic sheets. PIER B 29:355-392.

Rădulet, R., \& I. R. Ciric. 1971. Generalized functions in the theory of fields. Rev. Roum. Sci. Techn. Électrotechn. et Énerg. 16:565-591.

Scharstein, R. W. 1991. Helmholtz decomposition of surface electric current in electromagnetic scattering problems. In Proceedings of the 23rd Southeastern Symposium on System Theory, 424426. Columbia, SC: IEEE.

Schwartz, L. 1950. Théorie des distributions (Theory of distributions). Paris: Hermann. 
Schwartz, L. 2008. Mathematics for the physical sciences, chap. 2. New York: Dover (English translation Schwartz, L. 1965. Méthodes mathématiques pour les sciences physiques. Paris: Hermann).

Sprössig, W. 2010. On Helmholtz decompositions and their generalizations-an overview. Math. Meth. Appl. Sci. 33:374-383.

Stokes, G. G. 1849. On the dynamical theory of diffraction. Trans. Cambridge Phil. Soc. 9:1-62.

Tai, C. T. 1992. Generalized vector and dyadic analysis. New York: IEEE Press.

Taylor, J. G. 1956. Classical electrodynamics as a distribution theory. Math. Proc. Cambridge Phil. Soc. 52:119-134.

Taylor, J. G. 1958. Classical electrodynamics as a distribution theory. II. Math. Proc. Cambridge Phil. Soc. 54:258-264.

Van Bladel, J. 1960. On Helmholtz's theorem in multiply-bounded and multiply-connected regions. J. Franklin Inst. 269:445-462.

Van Bladel, J. 1993a. A discussion of Helmholtz' theorem. Electromagnetics 13:95-110.

Van Bladel, J. 1993b. A discussion of Helmholtz' theorem on a surface. AEÜ 47:131-136.

Van Bladel, J. 1993c. Three dimensional operators acting on surface sources. Radio Sci. 28: 841-845.

Van Bladel, J. 1995. Singular electromagnetic fields and sources. New York: IEEE Press.

Van Bladel, J. 1999. On the distributional form of the curl. Microw. Opt. Technol. Lett. 23:97-100. Van Bladel, J. 2007. Electromagnetic fields. New York: IEEE Press.

Vladimirov, V. S. 2002. Methods of the theory of generalized functions. London: Taylor \& Francis. Weatherburn, C. E. 1955. Differential geometry of three dimensions, chap. 12. Cambridge, UK: Cambridge University Press.

Zhou, X. L. 2007. On Helmholtz's theorem and its interpretations. JEMWA 21:471-483. 